\title{
The Properties of Open-Ended Meander Slot Antenna and its Applications to Antenna Design
}

\author{
† Young-soon Lee \\ + School of Electronic Eng, Kumoh Natl. Institute of Tech, 1 Yangho-dong, Kumi, Kyungbuk, 730-701, Korea
}

\begin{abstract}
The various properties such as the electric current distribution, resonant frequency and radiation patterns of open-ended meander slot antenna placed on a small ground plane, are investigated to give the help in performing antenna design. Based upon these characteristics, the designed antenna which is only $22 \mathrm{~mm}\left(0.06 \lambda_{g}\right)$ in height and $31 \mathrm{~mm}\left(0.09 \lambda_{g}\right)$ in width can operate at the $433 \mathrm{MHz}$ with the measured radiation efficiency of $8 \%$ and end-fire radiation pattern. These properties make the antenna suitable for the handheld device such as the wireless remote controller.
\end{abstract}

Key words : small ground plane, microstrip line feed, slot antenna, meander slot, end-fire radiation

\section{Introduction}

Microstrip antennas are being increasingly used in wireless handheld device because they have a number of advantages such as being light-weight, compact and conformal. Recently there has been increased interest in minimizing microstrip antennas for their applications in small wireless communication device. It is well known that microstrip slot antenna have the advantage of being able to produce bidirectional and unidirectional radiation patterns with somewhat large bandwidth in comparison with the other microstrip antennas. In order to reduce the size of these microstrip slot antenna, a few techniques based upon meander-type slot(Lancaste, 1999; Lu, 1998; Emson, 2000; Kim, 2001) were proposed. Recently, compact meander slot antenna with open-end(Cho, 2007) by which its overall size can be more reduced in comparison with the conventional meander slot antenna(Lancaste, 1999; Lu, 1998; Emson, 2000; Kim, 2001) was proposed. This reduction of antenna size is thought to be mainly due to the introduction of open -end which can be regarded as the magnetic wall. Besides of its reduction, the antenna with open-end has the end-fire radiation pattern (i.e., the radiation pattern of direction parallel to ground plane) due to the open-end as well as the radiation pattern of direction normal to ground plane, while the conventional antenna without open-end(Lancaste, 1999; Lu, 1998; Emson, 2000; Kim, 2001) have only the radiation pattern of direction normal to ground plane.

In the present study, in order to verify whether the open- end of the antenna proposed in Cho(2007) functions as the magnetic wall or not, electric current distribution on the ground plane in which meander slot is etched is investigated. And the various characteristics of the openended meander slot antenna placed on a small ground plane such as resonant frequencies and radiation patterns are presented. For instance, it is applied to the antenna design at $433 \mathrm{MHz}$ which corresponds to the $400 \mathrm{MHz}$ ISM band frequency.

\section{Properties of the proposed antenna}

Fig 1 shows the geometry of the proposed (open-ended meander slot) antenna. The meander slot open-terminated at its ends as shown in Fig. 1(a) where the line $\mathrm{A}-\mathrm{A}^{\prime}$ can be regared as the magnetic wall(Cho, 2007) is etched on the top ground plane of substrate. The microstrip feed line, which is L-shaped as shown in Fig. 1(b) to achieve the impedance matching and decrease the overall size at the same time, is proximately coupled into the slot. In order to check whether the line $\mathrm{A}-\mathrm{A}^{\prime}$ funtions as the magnetic wall or not, properties of the proposed antenna such as the electric current distribution, return loss and radiation pattern are investigated by simulations in comparison with a conventional one. The simulations were carried out on a package software MWS of [7] from Computer Simulation Technology(CST), based upon the design parameters presented in Cho(2007).

† Corresponding author, yslee@kumoh.ac.kr 054)478-7501 


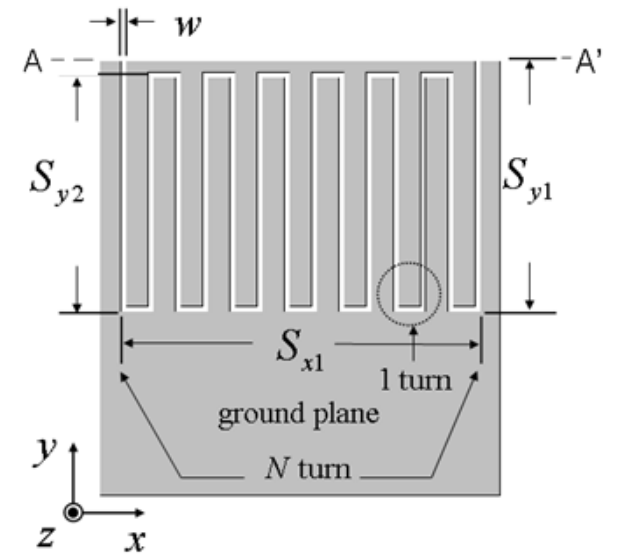

(a) Top view of the open-ended meander slot

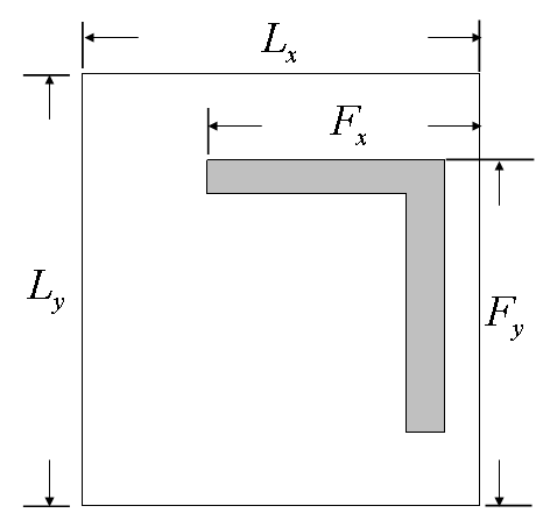

(b) Bottom view of feeding structure

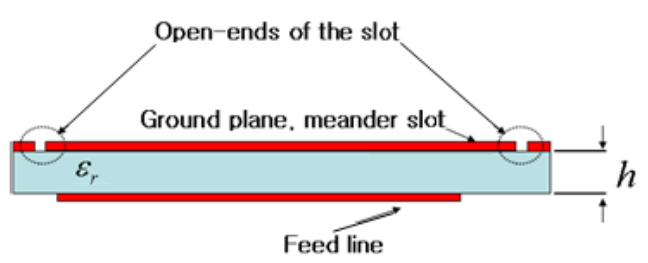

(c) Side view of the proposed antenna

Fig. 1 Geometry of the proposed (open-ended meander slot) antenna

The simulated electric current distribution on the ground plane of the proposed antenna is shown in Fig. 2(a), while that of the conventional meander antenna with twice long slot in which the line $\mathrm{A}^{-} \mathrm{A}^{\prime}$ is assumed as the magnetic wall(i.e., virtual open) is shown in Fig. 2(b). As shown in Fig 2(a), most of electric current is constrained along the circumference of the meander slot and its maximum value exists at the middle of the meander slot, while its value is extremely weak at the two open-ends of the meander slot due to the open termination. As shown in Fig 2(b), the electric current distribution of the conventional antenna is not only similar to that of the proposed antenna, but also it has even symmetry about the center line $\mathrm{A}-\mathrm{A}^{\prime}$ as well as a current null along the line(i.e., virtual open property). Consequently, it is obvious that the proposed antenna funtions as meander slot antenna type from the viewpoint of these eletric current ditributions. And also the horizontal lines $\mathrm{A}^{-} \mathrm{A}^{\prime}$ of the proposed antenna in Fig. 2(a) can be regarded as the magnetic wall due to the open termination, as if the horizontal lines $\mathrm{A}^{-} \mathrm{A}^{\prime}$ in Fig. 2(b) can be regarded as the magnetic wall due to the virtual open as mentioned above.

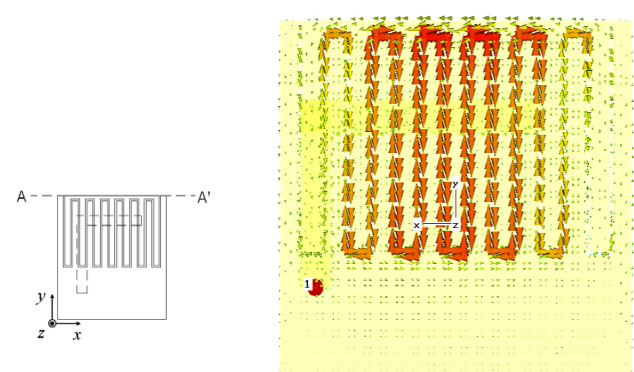

(a) Configuration of the proposed antenna and it's simulated current distribution

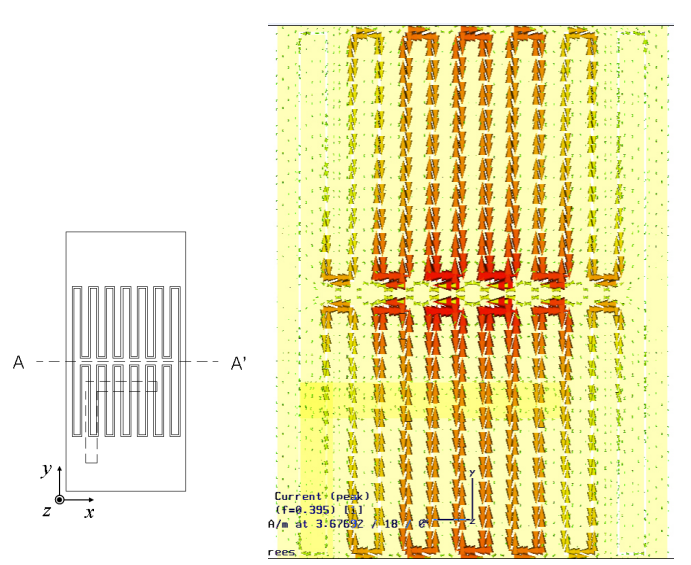

(b) Configuration of the conventional one which is twice as large as the proposed antenna and it's simulated current distribution

Fig. 2 Simualted electric current distribution on the ground plane

The simulated return loss for the proposed antenna and for the conventional antenna are shown in Fig 3(a) and Fig 3(b) respectively. It can be seen that two antennas have relatively similar resonant frequencies. It should be noted here that even though overall size of the proposed antenna is reduced to one half in comparison with the conventional antenna, its resonant frequency(405 $\mathrm{MHz})$ is relatively similar to that $(387 \mathrm{MHz})$ of the conventional antenna. 


\section{Young-soon Lee}

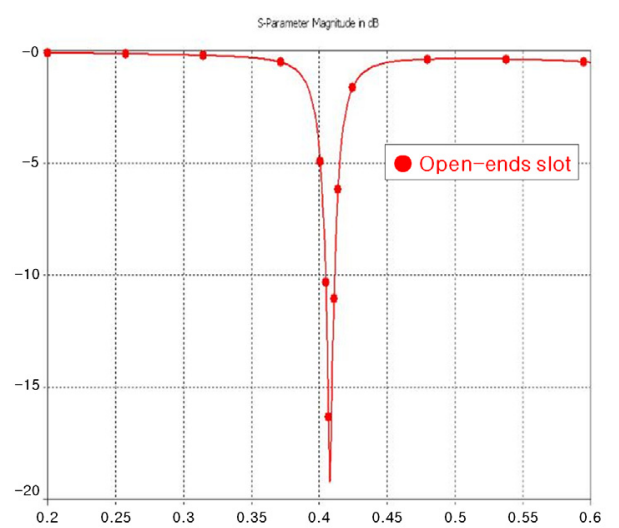

(a) for the proposed antenna of Fig. 2(a)

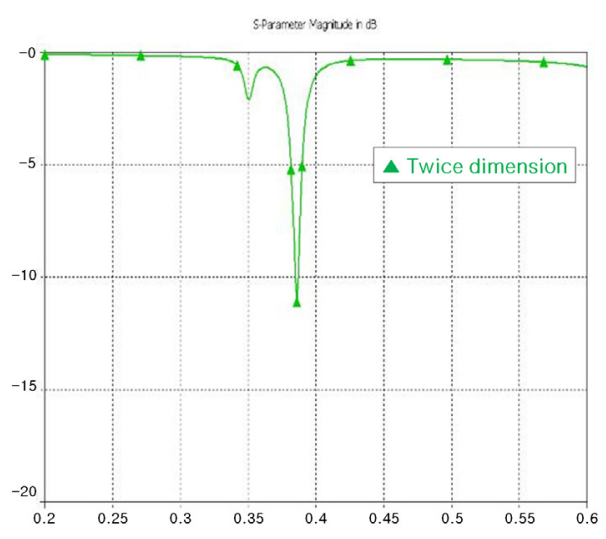

(b) for the conventional antenna of Fig. 2(b)

Fig. 3 Simulated return loss

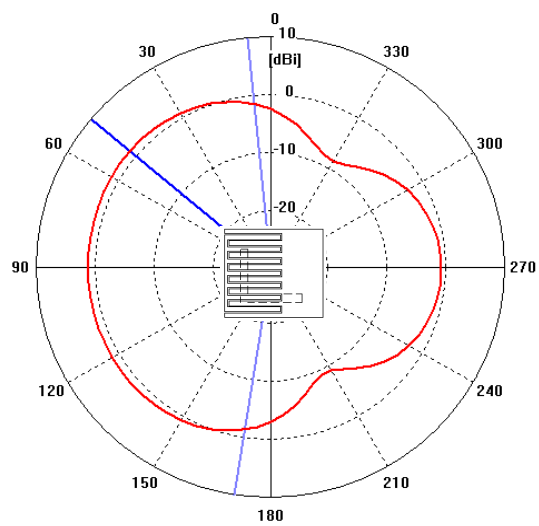

(a) $\mathrm{X}-\mathrm{Y}$ plane

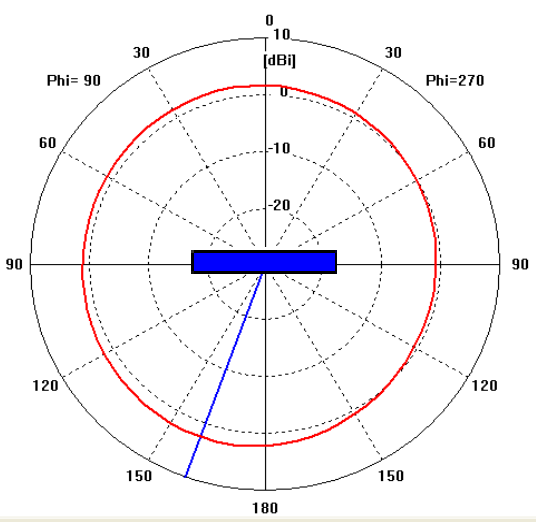

(b) $\mathrm{Y}-\mathrm{Z}$ plane

Fig. 4 Simulated radiation pattern for the proposed antenna of Fig. 2(a)

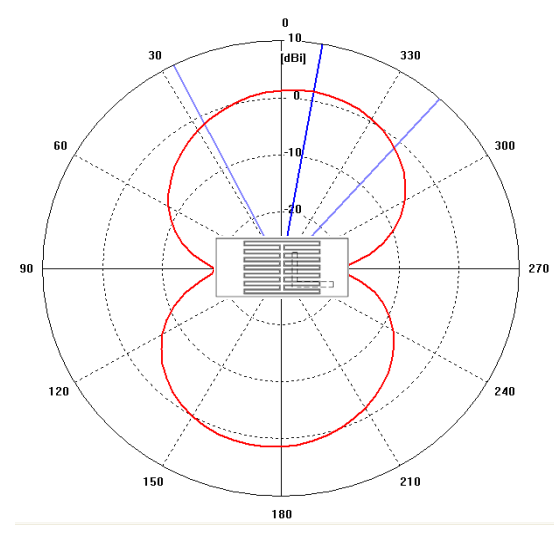

(a) $\mathrm{X}-\mathrm{Y}$ plane

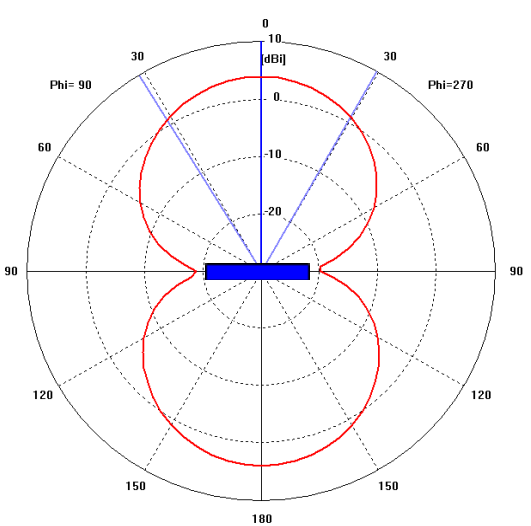

(b) $\mathrm{Y}-\mathrm{Z}$ plane

Fig. 5 Simulated radiation pattern for the conventional antenna of Fig. 2(b)

And also the simulated radiation pattern of the proposed antenna and the conventional antenna are shown in Fig 4 and Fig 5 respectively. The main difference between them is that the radiation pattern of the proposed antenna has the maximum value at $\mathrm{x}$-axis(i.e., end-fire radiation property), while that of the conventional antenna has null point at $\mathrm{x}$ axis as if the conventional meander slot antenna without open-ends should have null point always on the horizontal $(\mathrm{x}-\mathrm{y})$ plane. 


\section{Antenna design}

The proposed antenna is applied to a wireless communication system which is operated at $433 \mathrm{MHz}$. A copper meander slot antenna was fabricated on the FR-4 dielectric substrate with thickness $1.572 \mathrm{~mm}$ and relative dielectric constant 4.2. To obtain the optimum parameter of the proposed antenna at $433 \mathrm{MHz}$, simulations were also performed with the MWS throughout design process. It is observed that the resonant frequencies become low as the total lengths of slot become long. And also the variation of the length of feed line affects the return loss, while it dose not affect significantly the variation of resonant frequencies. Base upon these simulated results, the optimized values of each physical dimension of the proposed antenna are shown in Table 1.

Table 1 Design parameters

\begin{tabular}{|c|c||c|c|}
\hline parameters & values $[\mathrm{mm}]$ & parameters & values [mm] \\
\hline$L_{x}$ & 35 & $F_{x}$ & 17.5 \\
\hline$L_{y}$ & 38 & $F_{y}$ & 30.5 \\
\hline$S_{x 1}$ & 31 & $w$ & 0.35 \\
\hline$S_{y 1}$ & 22 & $h$ & 1.572 \\
\hline$S_{y 2}$ & 21 & $N$ & 11 [turn] \\
\hline
\end{tabular}

Fig 6 shows the simulated resonant frequency versus the number of meander slot turn with the slot width as a parameter. This means that the resonant frequency can be slightly changed by changing the slot width under the constant number of meander slot turn. The resonant frequency becomes low as the slot width becomes narrow. This result may be very useful practically in the fine tuning of the resonant frequency.

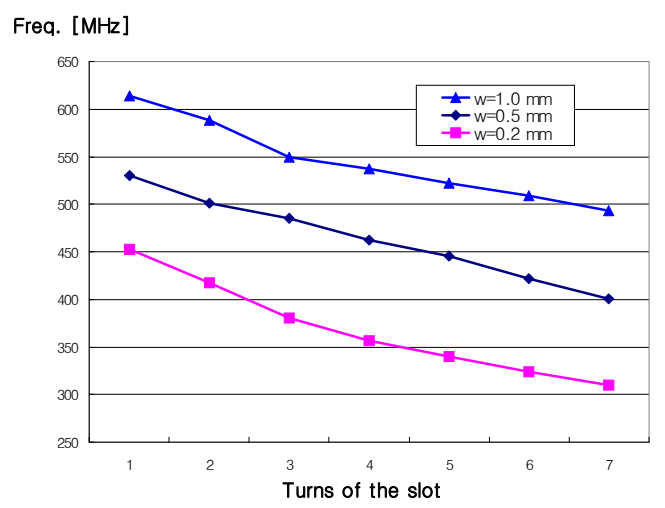

Fig. 6 Resonant frequency vs. number of meander slot turn with the slot width as a parameter
Figure 7 shows the photograph of the fabricated antenna. The return loss of the antenna has been measured using Agilent E8358A network analyzer. The simulated and measured return loss of the proposed antenna, as a function of frequency, are illustrated in Figure 8. It can be seen that the agreement between the simulated and measured results is fairly good.

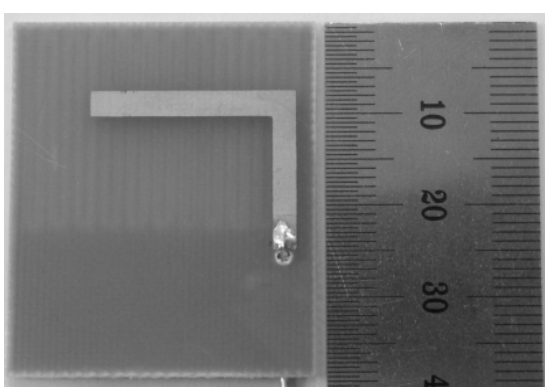

(a) Bottom view of feeding structure

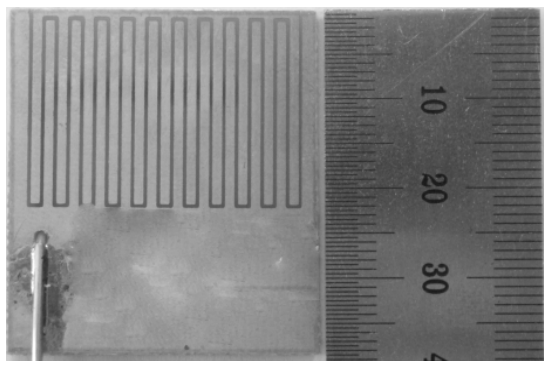

(b) Top view of the open-ended meander slot etched on the ground surface

Fig. 7 Fabricated antenna

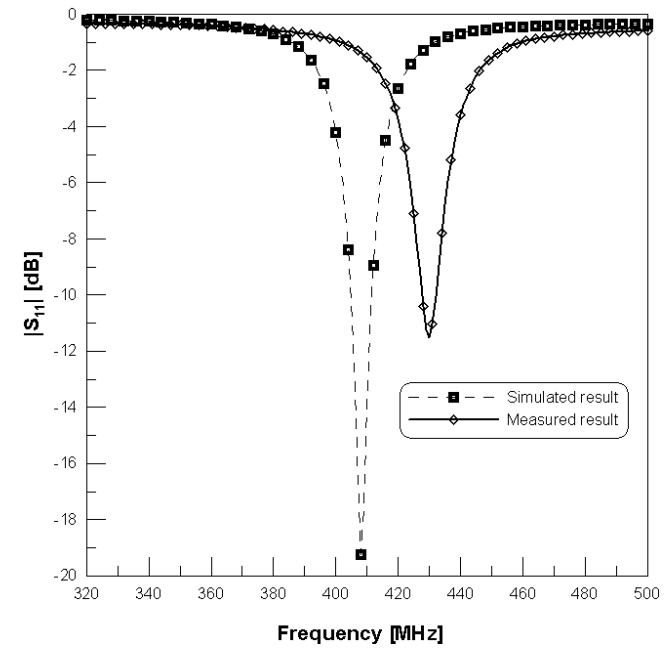

Fig. 8 Simulated and measured return loss of the fabricated antenna

The simulated and measured radiation patterns of the fabricated antenna are shown in Fig 9, where the solid lines and the dashed line are the simulated and measured results 


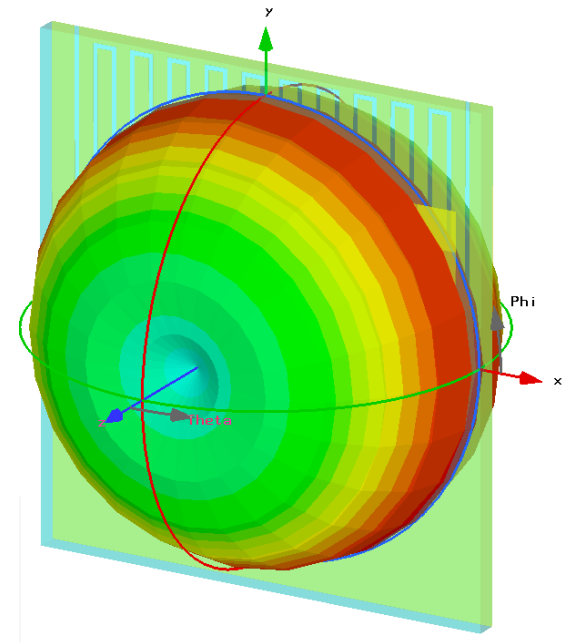

(a) 3-D radiation pattern

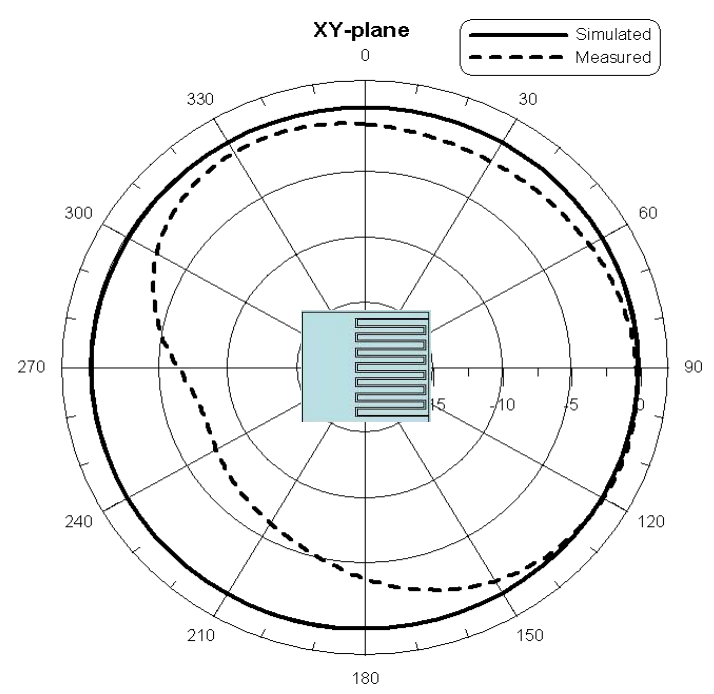

(b) Horizontal $(\mathrm{X}-\mathrm{Y})$ plane

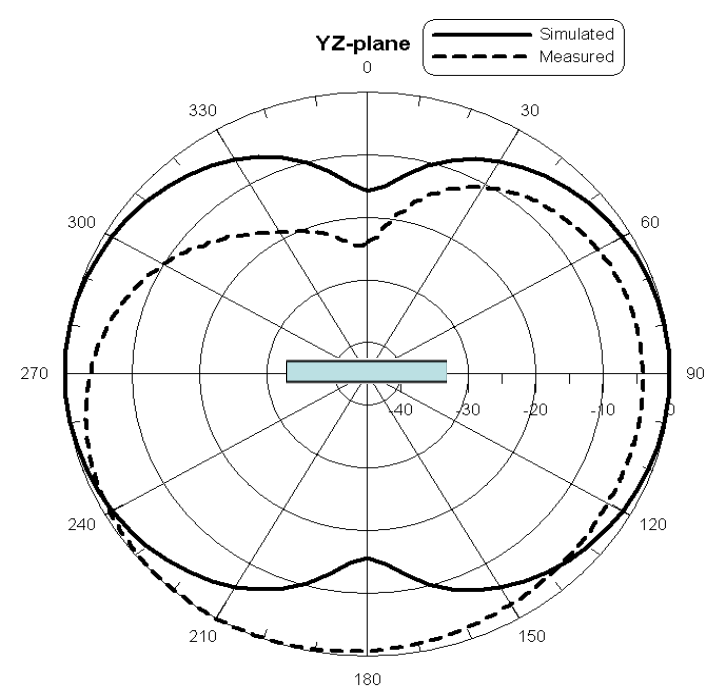

(c) Vertical $(Y-Z)$ plane

Fig. 9. Simulated and measured radiation patterns for fabricated antenna respectively. It is interesting that the maximum value of radiation field appears on the horizontal $(\mathrm{X}-\mathrm{Y})$ plane as shown in Fig 9(a) in contrast with the conventional antennas for which the null point appears always on the plane. The radiation efficiency measured by using the technique presented in [6] is about 8\% for the chosen substrate and dimensions of the meander slot. This value is relatively larger than that of the conventional meander slot antenna(Emsson, 1990; Kim, 2001).

\section{Conclusion}

A compact microstrip-fed open-ended meander slot antenna has been proposed and implemented. As a result, two main differences are observed when compared with the conventional meander slot antenna. First, since the openend can be regarded as the magnetic wall, the overall size of the meander slot antenna can be reduced about to one half than the conventional one. It should be noted that even though dimensions of the fabricated antenna are only 22 $\mathrm{mm} \times 31 \mathrm{~mm}\left(0.06 \lambda_{g} \times 0.09 \lambda_{g}\right)$, it can operate at $433 \mathrm{MHz}$. Second, the maximum value of the radiation field appears on the horizontal plane in contrast with the conventional one. Therefore the antenna may be very useful in a handheld electronic device such as the $433 \mathrm{MHz}$ wireless remote controller that needs the maximum radiation to the horizontal direction as a transmitter.

\section{Acknowledgements}

This paper was supported by Research Fund, Kumoh National Institute of Technology

\section{References}

[1] Cho, Y.K., Jung, H.H., Kim, E.J. and Lee, Y.S.(2007), "Compact meander slot antenna with open-ends", 2007 Korea-Japan Microwave Conference, TH04-2, Naha, Okinawa, Japan.

[2] Emson, C., Lancaster, M.J., Simkin, J., and Wang, H.Y.(2000), "Compact meander slot antennas", MICROWAVE AND OPTICAL TECHNOLOGY LETTERS, pp. 377 380 .

[3] Kim, J.M., Kim, K.W., Park, H.K. and Yook, J.G(2001), "Compact stripline-fed meander slot antenna", IEE Electronics Letters, pp. 995 996.

[4] Lancaste, M.J. and Wang, H.Y.(1999), “Aperture-coupled 
The Properties of Open-Ended Meander Slot Antenna and its Applications to Antenna Design

thin-film Superconducting meander antennas", IEEE

Transaction Antennas and Propagation, Vol. 47, pp. 829-836.

[5] Lu, J.H. and Wong, K.L.(1998), "Slot-loaded, meandere rectangular microstrip antenna with compact dual frequency operation”, Electronics Letters, Vol. 34, pp. $1048 \sim 1050$.

[6] Smith, G.S.(1977), "An analysis of the Wheeler method for measuring the radiation efficiency of antennas", IEEE Trans, AP-25, pp. 552 556.

[7] Trade mark of Computer Simulation Technology(CST).

Received 11 August 2011

Revised 28 September 2011

Accepted 28 September 2011 